Pacific Journal of Mathematics

PERMUTATIONS AS PRODUCTS OF CONJUGATE INFINITE 


\title{
PERMUTATIONS AS PRODUCTS OF CONJUGATE INFINITE CYCLES
}

\author{
EDWARD A. Bertram
}

Let $S=\left\{a_{i}\right\}_{-\infty}^{\infty}$ be a countable set and $P$ any permutation of $S$ with infinite support. Since the subgroup generated by the conjugacy class $\mathscr{K}$ of $P$ must be normal in $\operatorname{Sym}(S)$, we know that every permutation of $S$ is a product of permutations from $\mathscr{K}$. Since it has recently been discovered that every even permutation in the finite symmetric group $\operatorname{Sym}(n)$ may be expressed as a product of exactly two $n$-cycles, we are naturally led to a similar question for $\operatorname{Sym}(S)$ and the infinite cycle $C=\left(\cdots, a_{-2}, a_{-1}, a_{0}, a_{1}, a_{2}, \cdots\right)$, with support all of $S$. In this paper it is proved that for each $k \geqq 3$ every permutation of $S$ is a product of exactly $k$ cycles conjugate to $C$, but that no odd finite permutation is a product of two.

1. Introduction and notation. In 1951 Oystein Ore [4] proved that if $S$ is any infinite set, then every permutation $P$ of $S$ is a commutator, $P=Q\left(R Q^{-1} R^{-1}\right)$, of permutations $Q, R$. Throughout his constructions, the conjugacy class to which $Q$ belongs depends upon $P$. Allan Gray, in his dissertation [2], showed that this dependency of the class of $Q$ upon that of $P$ may be dropped. Our interest here is in the group $\operatorname{Sym}(\boldsymbol{S})$ of all permutations of a countable set $\boldsymbol{S}$. Gray showed that if $\mathscr{K}$ is the conjugacy class of permutations whose disjoint cycle decomposition consists of an infinite number of infinite (and no other) cycles, then $\operatorname{Sym}(\boldsymbol{S})=\mathscr{K} \mathscr{K}$, that is every permutation is a product of exactly two such conjugate permutations.

Recently, in [1], we studied the finite symmetric group Sym $(n)$, and solved the problem of characterizing those integers $l \leqq n$ for which every even permutation of $n$ symbols may be represented as a product of two (not necessarily disjoint) $l$-cycles. In particular, we showed that every even permutation can be expressed as a product of two $n$-cycles. (See also A. M. Gleason in [3, p. 172].)

In this paper we make use of the last result in considering a similar question for the group $\operatorname{Sym}(S), S$ countable: Let $\mathscr{C}_{\infty}$ denote the conjugacy class of infinite cycles, each of which moves each symbol of $\boldsymbol{S}$. Is there an integer $m$ such that every permutation in $\operatorname{Sym}(\boldsymbol{S})$ can be represented as a product of $m$ permutations, each from $\mathscr{C}_{\infty}$ ? If so, what is the smallest such $m$ ? We are able to show that $\operatorname{Sym}(\boldsymbol{S})=\mathscr{C}_{\infty}^{k}$ for $k \geqq 3$, but that $\operatorname{Sym}(\boldsymbol{S}) \neq \mathscr{C}_{\infty} \mathscr{C}_{\infty}$. To accomplish this, we prove that every infinite cycle $C$ may be represented as a product of two infinite cycles, where each cycle of the 
product moves those and only those symbols moved by $C$. Our construction is thus simpler than Ore's, who first showed that $C$ is a commutator by constructing $C$ as a product of two permutations, each the (disjoint) product of an infinite number of infinite cycles.

Let $\operatorname{Alt}(\boldsymbol{S})$ denote the subgroup of finite even permutations; $\mathscr{B}$ denotes the subset of permutations with one infinite cycle, and no restrictions on finite cycles; $\mathscr{E}$ denotes the subset of permutations with infinitely many 2-cycles and no infinite cycles or cycles of length $\geqq 4$. Then $\operatorname{Alt}(\boldsymbol{S}) \cup \mathscr{B} \cup \mathscr{E}$ is shown to be a subset of $\mathscr{C}_{\infty}^{2}$. The remainder of the proof consists in showing that if $P \notin \operatorname{Alt}(\boldsymbol{S}) \cup \mathscr{B} \cup \mathscr{E}$ then either $P Q \in \operatorname{Alt}(S) \cup \mathscr{B} \cup \mathscr{E}$ for some $Q \in \mathscr{C}_{\infty}$ or $P Q \in \mathscr{C}_{\infty}$ for some $Q \in \operatorname{Alt}(\boldsymbol{S}) \cup \mathscr{B} \cup \mathscr{E}$.

The subset of symbols of $\boldsymbol{S}$ which are moved by $P \in \operatorname{Sym}(\boldsymbol{S})$ is denoted by $M(P) .|M(P)|$ denotes the cardinality of $M(P),(P)_{n}$ denotes the cardinality of the set of cycles of length $n(\geqq 1)$ in the disjoint cycle decomposition of $P$, and $(P)_{\infty}$ denotes the cardinality of the set of infinite cycles in this decomposition. All products of permutations are executed from right to left. $P^{m}$ refers to the $m$ th iterate of $P$, for $m>0$, and the $-m$ th iterate of $P^{-1}$ (the inverse of $P$ ), for $m<0$.

2. Permutations with $M(P)$ finite. In this section we prove that $\operatorname{Alt}(\boldsymbol{S}) \subseteq \mathscr{C}_{\infty}^{2}$, but that no finite odd permutation of $\boldsymbol{S}$ belongs to $\mathscr{C}_{\infty}^{2} \cdot \mid$ denotes set theoretic difference.

THEOREM 2.1. Alt $(\boldsymbol{S}) \subseteq \mathscr{C}_{\infty}^{2}$.

Proof. Given $P \in$ Alt $(S)$, put $|M(P)|=l(<\infty)$. By Theorem 2 of [1], we know that the restriction of $P$ to $M(P)$ may be represented as a product $R S$ of two $l$-cycles $R$ and $S$, each moving precisely the symbols moved by $P$. If we fix $a \in M(P)$ and put $S \backslash M(P)=\left\{b_{i}\right\}_{1}^{\infty}$, we may list the symbols of $S$ in the manner displayed below:

$$
\cdots, b_{5}, b_{3}, b_{1}, a, S(a), S^{2}(a), \cdots, S^{l-2}(a), S^{-1}(a), b_{2}, b_{4}, b_{6}, \cdots
$$

Define two new permutations, $S^{*}$ and $R^{*}$, as follows:

$$
\begin{aligned}
& S^{*}\left(b_{2 m+1}\right)=b_{2 m-1} \\
& R^{*}\left(b_{2 m-1}\right)=b_{2 m+1} \\
& m \geqq 1 \\
& S^{*}\left(b_{1}\right)=a \\
& R^{*}(a)=b_{1} \\
& S^{*}\left(b_{2 m}\right)=b_{2 m+2} \\
& R^{*}\left(b_{2 m+2}\right)=b_{2 m} \\
& m \geqq 1 \\
& S^{*}\left(S^{-1}(a)\right)=b_{2} \\
& R^{*}\left(b_{2}\right)=R(a) \\
& S^{*}\left(S^{k}(a)\right)=S^{k+1}(a) \\
& R^{*}\left(S^{k+1}(a)\right)=R\left(S^{k+1}(a)\right) \\
& 0 \leqq k \leqq l-2 \text {. }
\end{aligned}
$$

It is now a straightforward verification that $R^{*}, S^{*} \in \mathscr{C}_{\infty}$ and $R^{*} S^{*}=$ $P$ on all of $S$. 
If we let $\mathscr{D}$ denote the set of all $P \in \operatorname{Sym}(\boldsymbol{S})$ with $|M(P)|<\infty$ and $P$ an odd permutation of $M(P)$, we have the following result [5].

\section{THEOREM 2.2. $\mathscr{D} \subseteq \operatorname{Sym}(S) \backslash \mathscr{C}_{\infty}^{2}$.}

Proof. By contradiction. Assume that there exists $P \in \mathscr{D}$ and $Q, R \in \mathscr{C}_{\infty}$ such that $P=Q R$. Since $R$ is an infinite cycle which moves each symbol of $\boldsymbol{S}$, we may rename the symbols of $\boldsymbol{S}$ with $\left\{x_{i}\right\}_{-\infty}^{\infty}$, where $R\left(x_{i}\right)=x_{i+1}$. Then there exists symbols, say $a$ and $c$, such that $a$ is the $x_{i} \in M(P)$ of smallest subscript, and $c$ is that of largest subscript. Since each symbol outside the "segment" $[a, c]$ is fixed by $P$, we know that $Q^{k}(a)=R^{-k}(a)$ and $Q^{-k}(R(c))=R^{k}(R(c))$, for $k \geqq 1$. Thus the list of symbols of $S$ can in fact be given by

$$
\cdots, Q^{3}(a), Q^{2}(a), Q(a), \alpha, R(a), R^{2}(a), \cdots, R^{-1}(c), c, R(c), R^{2}(c), \cdots .
$$

Note that $Q(R(c))$ must be among the symbols of the segment $[R(a)$, $\left.R^{-1}(c)\right]$, and that some symbols of the segment $\left[R(a), R^{-1}(c)\right]$ may be fixed by $P$. Permutations $Q^{*}$ and $R^{*}$ are next defined as follows:

$$
\begin{array}{ll}
Q^{*}(x)=Q(x) \text { for } x \in[R(a), R(c)] ; & R^{*}(x)=R(x) \text { for } x \in[a, c] \\
Q^{*}(a)=R(c) ; & R^{*}(R(c))=a \\
Q^{*}(x)=x \text { for } x \notin[a, R(c)] ; & R^{*}(x)=x \text { for } x \notin[a, R(c)] .
\end{array}
$$

Then $Q^{*} R^{*}=Q R=P$ everywhere on $S$. But, restricted to the set $[a, R(c)], Q^{*}$ and $R^{*}$ are each cycles of length $|M(P)|+1$, and on this set $Q^{*} R^{*}$ represents the restriction of $P$ to the set $[a, R(c)]$. This is a contradiction to the assumption that $P$ is an odd permutation on the set $[a, R(c)]$.

3. Permutations with $(P)_{\infty}=1$. We first prove that $\mathscr{C}_{\infty} \subseteq \mathscr{C}_{\infty}^{2} \subseteq$ $\mathscr{C}_{\infty}^{3} \subseteq \cdots$. This, together with Theorem 2.1, yields Alt $(S) \subseteq \mathscr{C}_{\infty}^{2} \cong$ $\mathscr{C}_{\infty}^{3} \subseteq \ldots$. Our construction also shows that every infinite cycle $C$ is a product of two permutations, each permutation in the conjugary class of $C$. The result is simpler than that of Ore [4, p. 309, Theorem 2], who showed that $C$ may be written as a product of two permutations, each an infinite (disjoint) product of infinite cycles.

Here we regard $S$ as the set $\boldsymbol{Z}$ of integers $\{\cdots,-3,-2,-1,0$, $1,2, \cdots\}$. Let $C$ denote the permutation of $\boldsymbol{Z}$ given by $C(i)=i+1$ for each $i \in \boldsymbol{Z}$. Then $\mathscr{C}_{\infty}$ is the conjugacy class of $C$.

THEOREM 3.1. $\mathscr{C}_{\infty} \subseteq \mathscr{C}_{\infty}^{2} \subseteq \mathscr{C}_{\infty}^{3} \subseteq \cdots$.

Proof. We need only show that $C \in \mathscr{C}_{\infty}^{2}$. For this, let $A=$ $(\cdots,-9,9,-6,6,-3,3,0,-1,2,1,-2,-4,4,-5,5,-7,7,-8,8, \cdots)$ 
and $B=(\cdots, 8,-9,7,-8,5,-6,4,-5,-2,0,2,-3,1,-, 1,3$, $-4,6,-7,9,-10, \cdots)$ be given by

$$
\begin{array}{lrl}
A(n)=-n, n \leqq-3 & B(n)=-(n+1), n \geqq 3 \\
\quad \text { and for } n \geqq 3: & \text { and for } n \leqq-3(n \neq-5): \\
A(n)=-(n-3), n \equiv 0(\bmod 3) & B(n)=-(n+2), n \equiv 0(\bmod 3) \\
A(n)=-(n+1), n \equiv 1(\bmod 3) & B(n)=-(n+3), n \equiv 1(\bmod 3) \\
A(n)=-(n+2), n \equiv 2(\bmod 3) & B(n)=-(n+2), n \equiv 2(\bmod 3)
\end{array}
$$

Obviously, $A \in \mathscr{C}_{\infty}$. For $n \notin\{-5,-2,-1,0,1,2\}$, by dividing the proof according to the residue class of $n(\bmod 3)$, it is quickly verified that $A B(n)=C(n)=n+1$; and this is immediate for $n \in\{-5$, $-2,-1,0,1,2\} . B$ is a permutation, and it follows that $B \in \mathscr{C}_{\infty}$, since for each $n \in \boldsymbol{Z}$ there exists an integer $k(n)$ such that $B^{k}(n)=0$.

\section{THEOREM 3.2. $\mathscr{B} \cong \mathscr{C}_{\infty}^{2}$.}

Proof. By Theorem 3.1, we may assume that $P \in \mathscr{B}$ satisfies $\sum_{n \geqq 1}(P)_{n}>0$. Initially, we suppose that $(P)_{1}=\mu>0$ and $\sum_{n \geqq 2}(P)_{n}=$ $\nu>0$, and if $P$ does not satisfy one (or both) of these it will be apparent what modifications must be made. Denote the set of 1cycles of $P$ by $\left\{\left(f_{j}\right) \mid 1 \leqq j\right\}$, and the set of cycles of length $\geqq 2$ by $\left\{\left(a_{i, 1} a_{i, 2} a_{i, 3} \cdots a_{i, l(i)}\right) \mid 1 \leqq i\right\}$. Either set may be infinite, of course. For each set $\left\{a_{i, j}\right\}_{j=1}^{l(i)}$ we introduce a new set $\left\{b_{i, j}\right\}_{j=1}^{l(i)-1}$ and let $S$ be the set of symbols

$$
\begin{aligned}
& \boldsymbol{Z} \cup \bigcup_{i \geqq 1}\left\{a_{i, 1}, a_{i, 2}, a_{i, 3}, \cdots, a_{i, l(i)}\right\} \cup \bigcup_{j \geqq 1}\left\{f_{j}\right\} \\
& \cup \bigcup_{i \geqq 1}\left\{b_{i, 1}, b_{i, 2}, \cdots, b_{i, l(i)-1}\right\}
\end{aligned}
$$

where the infinite cycle of $P$ is now to be given by

$$
\begin{aligned}
& \left(\cdots,-2,-1,0,1,2,3, b_{1,1}, b_{1,2}, \cdots, b_{1, l(1)-1}, 4,5,6, b_{21},\right. \\
& \left.b_{2,2}, \cdots, b_{2, l(2)-1}, \cdots\right) .
\end{aligned}
$$

In general, the sequence $b_{i, 1}, b_{i, 2}, \cdots, b_{i, l(i)-1}$ is placed between the integers $3 i$ and $3 i+1,1 \leqq i$.

Modify the infinite cycle $A$ of the preceding theorem by placing $f_{i}$ between $-3 i$ and $3 i$, and by placing the alternating sequence $a_{i, 1}, b_{i, 1}, a_{i, 2}, b_{i, 2}, \cdots, b_{i, l(i)-1}, a_{i, l(i)}$ between $-(3 i+1)$ and $3 i+1$.

$$
\begin{aligned}
A= & \left(\cdots, 9,-6, f_{2}, 6,-3, f_{1}, 3,0, \cdots,-4, a_{1,1}, b_{1,1}, a_{1,2}, \cdots, b_{1, l(1)-1},\right. \\
& \left.a_{1, l(1)}, 4,-5,5,-7, a_{2,1}, b_{2,1}, \cdots, a_{2, l(l)}, 7,-8,8,-11, \cdots\right) .
\end{aligned}
$$

Furthermore, let 


$$
\begin{aligned}
B= & \left(\cdots,-8,5, f_{2},-6, \cdots, 2, f_{1},-3, \cdots, 3, a_{1,1}, b_{1,1}, a_{1,2}, \cdots,\right. \\
& \left.b_{1, l(1)-1}, a_{1, l(1)},-4,6, a_{2,1}, b_{2,1}, \cdots, a_{2, l(2)},-7,9, \cdots\right),
\end{aligned}
$$

which is now an obvious modification of the $B$ in the preceding theorem. We see that $A, B \in \mathscr{C}_{\infty}$; and $P=A B$ needs only a straightforward verification.

\section{CoROLlaRY 3.2.1. $\operatorname{Alt}(S) \cup \mathscr{B} \cup \mathscr{D} \subseteq \mathscr{C}_{\infty}^{3} \subseteq \mathscr{C}_{\infty}^{4} \subseteq \cdots \cdot$}

Proof. By Theorems 2.1, 3.1 and 3.2, Alt $(S) \cup \mathscr{B} \subseteq \mathscr{C}_{\infty}^{2} \subseteq \mathscr{C}_{\infty}^{3} \subseteq \cdots$. To prove that $\mathscr{D} \subseteq \mathscr{C}_{\infty}^{3}$, again let $S=Z$, and suppose $P \in \mathscr{D}$. Then there exists a $Q \in \operatorname{Alt}(S)$ such that $P=Q \circ(01)$. By Theorem 2.1, $Q=A B, A, B \in \mathscr{C}_{\infty}$. Hence $P=A B \circ(01)$. If either $B^{k}(0)=1$ or $B^{k}(1)=0, k \geqq 1$, then $B \circ\left(\begin{array}{ll}0 & 1\end{array}\right)$ consists of exactly one infinite cycle and one (disjoint) $k$-cycle. By Theorem 3.2, since $B \circ\left(\begin{array}{ll}0 & 1\end{array}\right)$ belongs to $\mathscr{B}, B \circ(01) \in \mathscr{C}_{\infty}^{2}$. But then $P \in \mathscr{C}_{\infty}^{3}$.

4. Permutations with infinitely many finite, and no infinite cycles. Let $\mathscr{E}=\left\{P \mid(P)_{2}=\infty,(P)_{n \geqq 4}=0\right\}$ and $\mathscr{F}=\left\{P \mid(P)_{\infty}=0\right.$, $\left.\sum_{n \geqq 1}(P)_{n}=\infty\right\}$. In Theorem 4.1 we prove that $\mathscr{F} \subseteq \mathscr{C}_{\infty}^{3}$. Of course $\mathscr{E} \subseteq \mathscr{F}$, so $\mathscr{E} \subseteq \mathscr{C}_{\infty}^{3}$, but in order to conclude that $\operatorname{Sym}(\boldsymbol{S}) \subseteq \mathscr{C}_{\infty}^{3}$, we will need the fact that $\mathscr{E} \subseteq \mathscr{C}_{\infty}^{2}$, and this is shown in Theorem 4.2 .

\section{THEOREM 4.1. $\mathscr{F} \subseteq \mathscr{C}_{\infty}^{3}$.}

Proof. Let $P \in \mathscr{F}$ be expressed as $P=\prod_{i=-\infty}^{\infty}\left(p_{i, 1} p_{i, 2} \cdots p_{i, l(i)}\right)$, where $S$ is now the collection $\left\{p_{i, j}\right\}$. Define the permutation $Q$ by $Q\left(p_{i, 1}\right)=p_{i+1,1},-\infty<i<\infty$, and $Q(x)=x$ for $x \notin\left\{p_{i, 1}\right\}_{-\infty}^{\infty}$. Then $Q=$ $\left(\cdots, p_{-21}, p_{-1,1}, p_{0,1}, p_{1,1}, p_{2,1}, \cdots\right)$ is in $\mathscr{B}$, and thus $Q \in \mathscr{C}_{\infty}^{2}$. But $Q P=$ $\left(\cdots, p_{-1,1}, p_{-1,2}, \cdots, p_{-1, l(1)}, p_{0,1}, p_{02}, \cdots, p_{0, l(0)}, \cdots\right)$ is in $\mathscr{C}_{\infty}$, and hence $P \in \mathscr{C}_{\infty}^{3}$.

\section{THEOREM 4.2. $\mathscr{E} \subseteq \mathscr{C}_{\infty}^{2}$.}

Proof. Let $P \in \mathscr{E} . \quad S$ is here considered as a sequence of blocks of symbols. On each block, mappings $R, S$ are defined, and when these restrictions are pieced together it will be seen that $P=R S$ and $R, S$ are permutations in $\mathscr{C}_{\infty}$. Let $\left(b_{1} b_{2}\right)\left(b_{3} b_{4}\right)$ and $\left(b_{4 i+1} b_{4 i+2}\right)\left(b_{4 i+3} b_{4(i+1)}\right)$, $i \geqq 1$, denote the infinitely many pairs of transpositions in the disjoint cycle decomposition of $P$. We begin by defining $R, S$ as follows: $S\left(b_{1}\right)=b_{3}, S\left(b_{3}\right)=b_{2}, S\left(b_{2}\right)=b_{4}, S\left(b_{4}\right)=b_{7}, S\left(b_{5}\right)=b_{1} ; R\left(b_{3}\right)=b_{2}, R\left(b_{2}\right)=b$, $R\left(b_{4}\right)=b_{1}, R\left(b_{7}\right)=b_{3}, R\left(b_{1}\right)=b_{6}$. The most complicated case is when there are 1-cycles and 3-cycles in $P$; for each $i \geqq 1$ for which there 
is a 1-cycle $\left(f_{i}\right)$ and a 3 -cycle $\left(c_{3 i-2} c_{3 i-1} c_{3 i}\right)$, the $i$ th block of $S$ is given by $b_{4 i+1}, b_{4 i+2}, f_{i}, b_{4 i+3}, b_{4(i+1)}, c_{3 i-2}, c_{3 i-1}, c_{3 i}$. With the exception of the first few $b_{j}$ noted above, we define $R$ and $S$ on such a block as in Figure 1, where parentheses have been inserted to single out the cycles of $P$.

\section{$\mathrm{R}$}

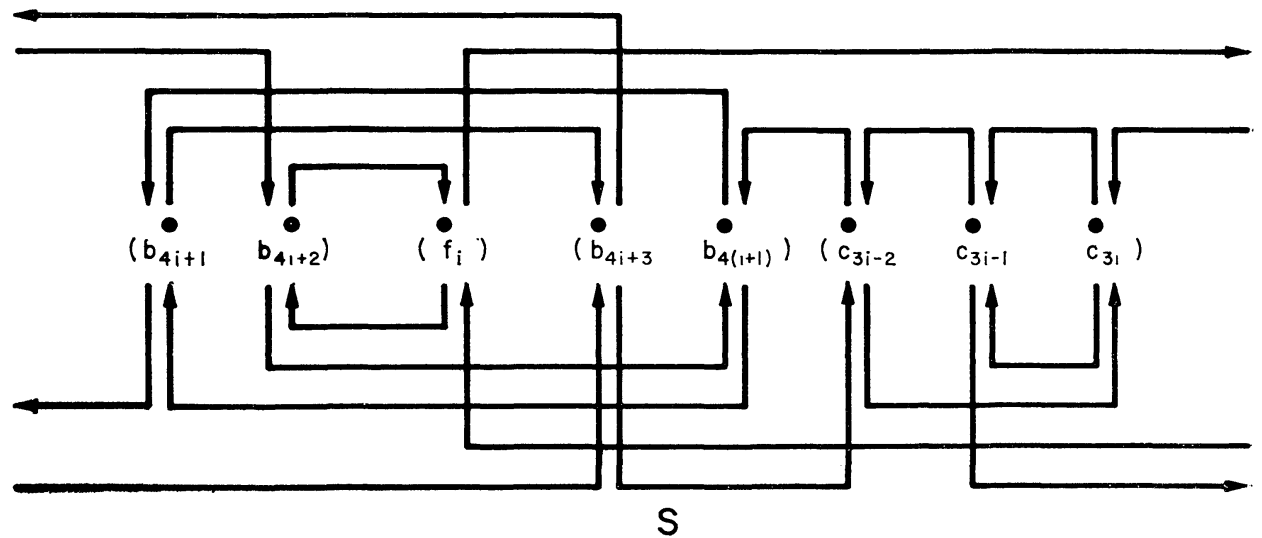

FIGURE 1.

All directed edges above the symbols refer to the mapping $R$ (e.g., $R\left(b_{4 i+1}\right)=b_{4 i+3}$ ), whereas those below the symbols refer to $S$ (e.g., $\left.S\left(b_{4(i+1)}\right)=b_{4 i+1}\right)$. If either or both of $(P)_{1},(P)_{3}$ is finite, there will eventually be blocks of symbols either without a 1-cycle, or 3-cycle, or both. In such cases, we simply delete the appropriate incoming and outgoing directed edges, and redefine $R, S$ in the obvious way.

We may now patch the restrictions together in the various cases. The second choice is given for $S$ and $R$ in case there is either no $i$ th 1-cycle, or no $i$ th 3 -cycle, or both.

$$
\begin{array}{crl}
S\left(b_{4(i+1)+1}\right) & =f_{i}, b_{4 i+2} & R\left(b_{4(i+2)}\right)=f_{i}, b_{4(i+1)+2} \\
S\left(b_{4 i+3}\right) & =c_{3 i-2}, b_{4(i+1)+3} & R\left(b_{4(i+1)+3}\right)=c_{3 i}, b_{4(i+1)} \\
S\left(c_{3 i-1}\right)=b_{4(i+1)+3} & R\left(f_{i}\right)=b_{4(i+1)+2} .
\end{array}
$$

In each case $R S=P$ on all of $S$, and $R, S \in \mathscr{C}_{\infty}$. Thus $P \in \mathscr{C}_{\infty}^{2}$.

5. Permutations with more than one infinite cycle. Let $\mathscr{A}$ denote the set of permutations $P$ of $S$ which satisfy $1<(P)_{\infty} \leqq \infty$. Then $\operatorname{Sym}(\boldsymbol{S})=\operatorname{Alt}(\boldsymbol{S}) \cup \mathscr{D} \cup \mathscr{B} \cup \mathscr{F} \cup \mathscr{A}$, and we have shown that the first four sets in this union are subsets of $\mathscr{C}_{\infty}^{3}$. We prove that $\mathscr{A} \subseteq \mathscr{C}_{\infty}^{3}$, in two parts, and will thus have proved that $\operatorname{Sym}(S)=\mathscr{C}_{\infty}^{k}$, for $k \geqq 3$. 


\section{THEOREM 5.1. $\left\{P \in \operatorname{Sym}(S) \mid 1<(P)_{\infty}<\infty\right\} \subseteq \mathscr{C}_{\infty}^{3}$.}

Proof. We divide the argument according to whether $(P)_{\infty}$ is odd or even, including more detail for the even case, since for $(P)_{\infty}$ odd the construction is very similar. Note that $P$ may have cycles of finite length. Let $S=\left\{a_{i, j}\right\} \cup\left\{b_{r, s}\right\}$, where the infinite cycles of $P$ are denoted by $\left(\cdots, a_{-1, j}, a_{0, j}, a_{1, j}, \cdots\right), 1 \leqq j \leqq m=(P)_{\infty}$ and the finite cycles by $\left(b_{r, 1} b_{r, 2} \cdots b_{r, l(r)}\right)_{r \geq 1}$. For purposes of the construction, the $\left\{a_{i, j}\right\}$ are realized as the (integral) lattice points $\{(i, j)\}$ in the horizontal strip of the plane given by $-\infty<i<\infty, 1 \leqq j \leqq m$; each $\left\{b_{r, s}\right\}_{s=1}^{\ell(r)}$ may be realized as any collection of $l(r)$ points on some $r$ th horizontal line below the horizontal axis.

Let $m\left(=(P)_{\infty}\right)$ be even, and define the mapping $Q$ on $\boldsymbol{S}$ as follows (see Figure 2a, where $m=6$ ):

$$
\begin{aligned}
& Q\left(a_{i, j}\right)=\left\{\begin{array}{l}
a_{i-1, j+1} \text { if } i \equiv j(\bmod 2) \text { and } j<m \\
a_{i-1, j-1} \text { if } i \neq j(\bmod 2) \text { and } 1<j
\end{array}\right. \\
& Q\left(a_{i, m}\right)=a_{-i+1, m} \text { if } i \equiv 0(\bmod 2) \\
& Q\left(a_{2 r, 1}\right)=b_{r, 1} \text { and } Q\left(b_{r, 1}\right)=a_{-2 r-1,1}, \text { if } l(r)=1 .
\end{aligned}
$$

If $l(r)>1$, put $Q\left(a_{2 r, 1}\right)=b_{r, l(r)}, Q\left(b_{r, j}\right)=b_{r, j-1}(2<j \leqq l(r)), Q\left(b_{r, 2}\right)=$ $a_{-2 r-1,1}, Q\left(a_{-2 r, 1}\right)=b_{r, 1}$, and $Q\left(b_{r, 1}\right)=a_{2 r-1,1}$. If there is no $r$ th |(finite)

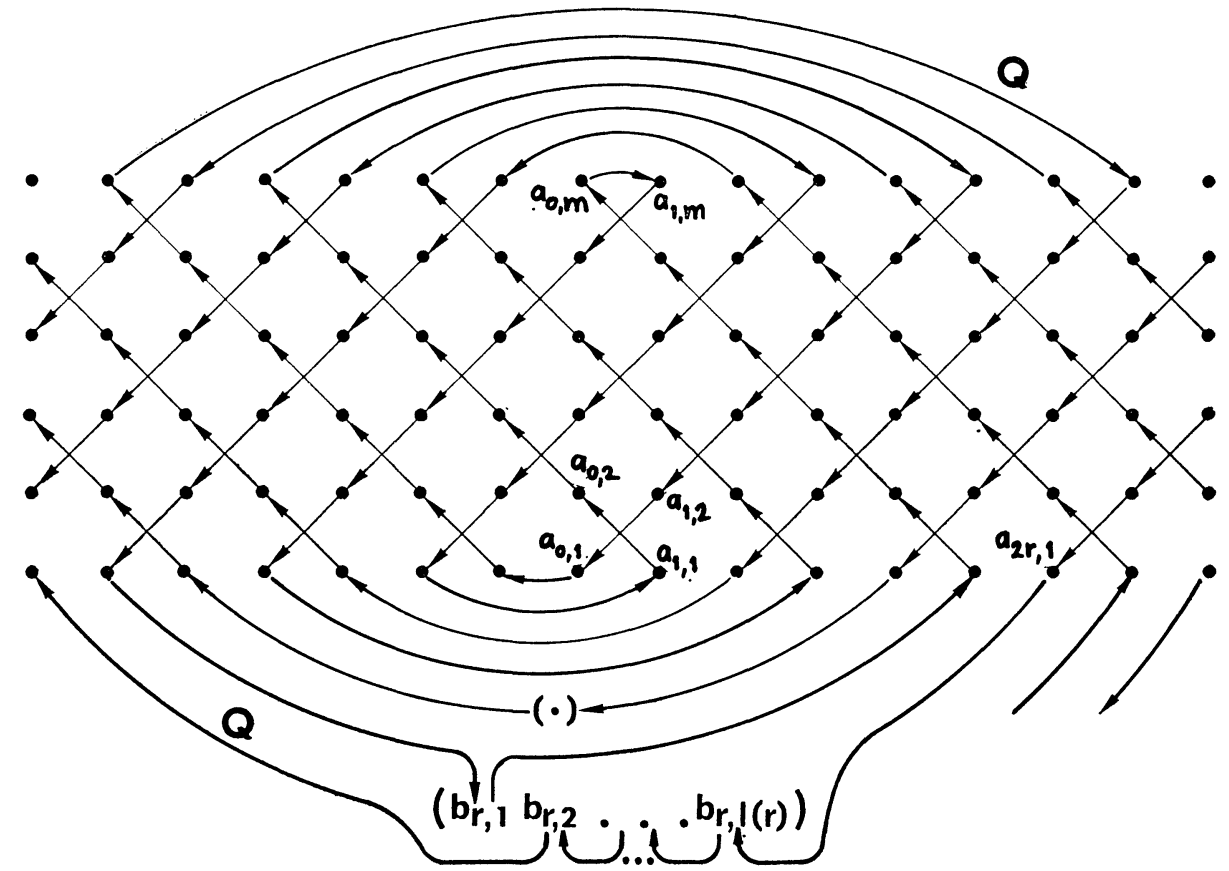

FIgURE 2a. $\quad m \equiv 0(\bmod 2)$ 
cycle of $P$, put $Q\left(a_{2 r, 1}\right)=a_{-2 r-1,1}$.

In all cases, $Q$ is a permutation in $\mathscr{C}_{\infty}$. Furthermore, whenever the $r$ th finite cycle of $P$ is a 1-cycle, $P Q$ contains the 3-cycle $\left(a_{2 r, 1} b_{r, 1} a_{-2 r, 1}\right)$. For each finite cycle of length $l(r) \geqq 2, P Q$ contains $l(r)-2$ 1-cycles (e.g., $P Q$ fixes $b_{r, j}, 2 \leqq j \leqq l(r)$ ) and two 2-cycles (e.g., $\left(a_{2 r}, b_{r, 1}\right)$ and $\left.\left(a_{-2 r, 1} b_{r, 2}\right)\right)$. In any case, $P Q$ contains the 2-cycles $\left(a_{i, j} a_{i, j+1}\right)$ for $i \equiv j(\bmod 2)$ and $1 \leqq j<m$, and the 2-cycles $\left(a_{i, m} a_{-i+2, m}\right)$ for $i \equiv 0(\bmod 2)$. Thus, no matter how many or how large the finite cycles of $P, P Q \in \mathscr{E}$. Since $\mathscr{E} \subseteq \mathscr{C}_{\infty}^{2}$ and $Q \in \mathscr{C}_{\infty}, P \in \mathscr{C}_{\infty}^{3}$.

If $(P)_{\infty}=m \equiv 1(\bmod 2)$, we may assume (by Theorems 3.1 and 3.2 ) that $m \geqq 3$, and realize the infinite cycles of $P$ as moving the appropriate lattice points, as in the even case. Define $Q$ on these lattice points as follows (see Figure $2 \mathrm{~b}$, where $m=5$ ):

$$
\begin{aligned}
& Q\left(a_{i, j}\right)=\left\{\begin{array}{l}
a_{i-1, j+1} \text { if } i \equiv j(\bmod 2) \text { and } j<m \\
a_{i-1, j-1} \text { if } i \neq j(\bmod 2) \text { and } 1<j
\end{array}\right. \\
& Q\left(a_{i, m}\right)=a_{3-i, m} \text { if } i \equiv 1(\bmod 2)
\end{aligned}
$$

If there is no $r$ th finite cycle of $P$, define $Q\left(a_{2 r, 1}\right)=a_{-2 r+1,1} \cdot Q$ is defined on the elements $\left\{b_{r, 1}, b_{r 2}, \cdots, b_{r, l(r)}\right\}$ of the $r$ th finite cycle, on $a_{2 r, 1}$ and $a_{-2(r-1), 1}$ just as in the even case, where $a_{-2(r-1), 1}$ now plays the role that $a_{-2 r, 1}$ did there. Again $Q \in \mathscr{C}_{\infty}, P Q$ contains at most 1-cycles, 2-cycles, and 3-cycles, $P Q \in \mathscr{C}_{\infty}^{2}$, and $P \in \mathscr{C}_{\infty}^{3}$.

\section{$P: a_{i, j} \rightarrow a_{i+1, j}$}

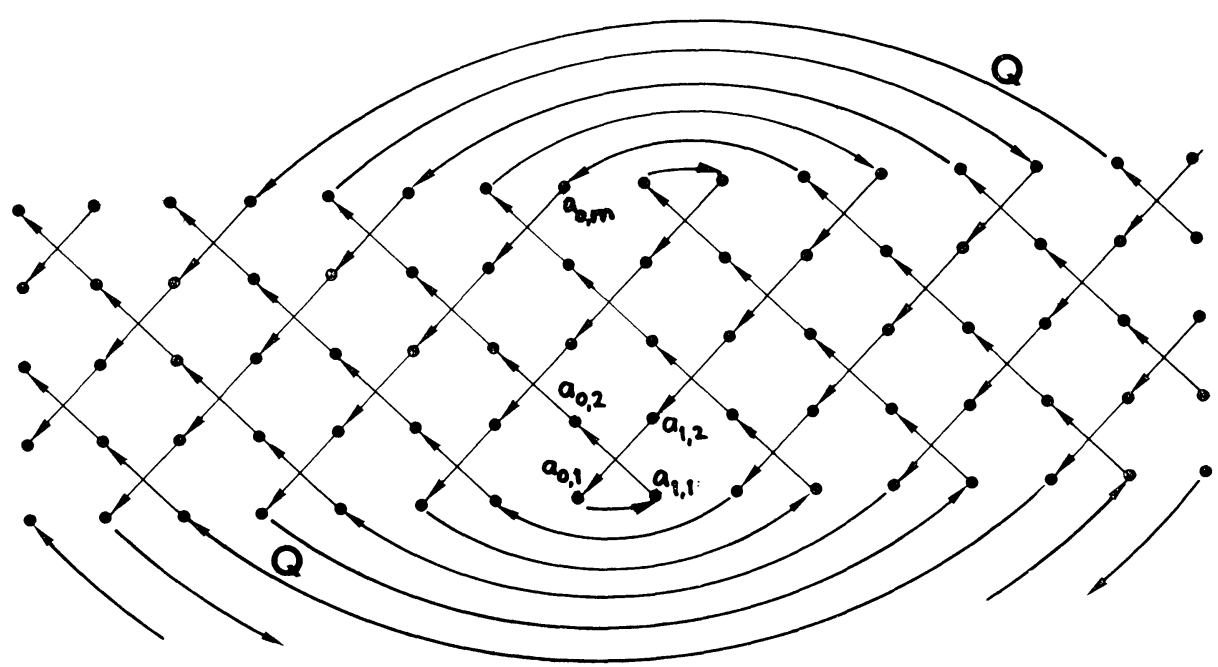

FIGURE $2 b . \quad m \equiv 1(\bmod 2)$ 


\section{THEOREM 5.2. $\left\{P \mid(P)_{\infty}=\infty\right\} \subseteq \mathscr{C}_{\infty}^{3}$.}

Proof. Again let $\boldsymbol{S}=\left\{a_{i, j}\right\} \cup\left\{b_{r, s}\right\}$, with the $a_{i, j}$ realized as the lattice points $(i, j)$ in the upper half plane, $1 \leqq j<\infty,-\infty<i<\infty$. The infinite cycles of $P$ are $\left(\cdots, a_{-1, j}, a_{0, j}, a_{1, j}, \cdots\right)$ for even $j$ and $\left(\cdots, a_{1, j}, a_{0, j}, a_{-1, j}, \cdots\right)$ if $j$ is odd (see Figure 3). In case $1 \leqslant \sum_{n \geqq 1}(P)_{n}$, the $b_{r, s}$ represent the symbols of the finite cycles of $P:\left\{\left(b_{r, 1} b_{r, 2} \cdots b_{r, l(r)}\right)\right\}_{r \geqq 1}$, and are realized as in the previous theorem.

If $(P)_{n \geqq 1}=0$, define the mapping $Q$ as in Figure 3 ; it is a straightforward verification that $Q \in \mathscr{C}_{\infty}$ and $P Q$ contains the 3-cycle $\left(a_{-1,1} a_{0,1} a_{1,2}\right)$, and otherwise only 2-cycles. Thus $P Q \in \mathscr{E} \subseteq \mathscr{C}_{\infty}^{2}$, and $P \in \mathscr{C}_{\infty}^{3}$. Otherwise, $1 \leqslant \sum_{n \geqq 1}(P)_{n}$, and we redefine $Q$ in nearly the same way as in the previous theorem, noting again that $Q$ remains in $\mathscr{C}_{\infty}:$ For $l(r)=1$, put $Q\left(a_{2 r, 1}\right)=b_{r, 1}$ and $Q\left(b_{r, 1}\right)=a_{-2 r, 1}$. For $l(r)>$ 1 , put $Q\left(a_{2 r, 1}\right)=b_{r, l(r)}, Q\left(b_{r, j}\right)=b_{r, j-1}$ for $2<j \leqq l(r), Q\left(b_{r, 2}\right)=a_{-2 r, 1}$, $Q\left(a_{-2 r-1,1}\right)=b_{r, 1}$ and $Q\left(b_{r, 1}\right)=a_{2 r+1,1}$. For each occurrence of a 1-cycle of $P$, say $\left(b_{r, 1}\right), P Q$ contains the 3 -cycle $\left(a_{2 r, 1} b_{r, 1} a_{-2 r-1,1}\right)$. For each finite cycle of length $l \geqq 2, P Q$ contains $l-2$ 1-cycles and one 2-cycle. Again $P Q$ contains at most 1-cycles, 2-cycles, and 3-cycles, $P Q \in \mathscr{C}_{\infty}^{2}$ and $P \in \mathscr{C}_{\infty}^{3}$.

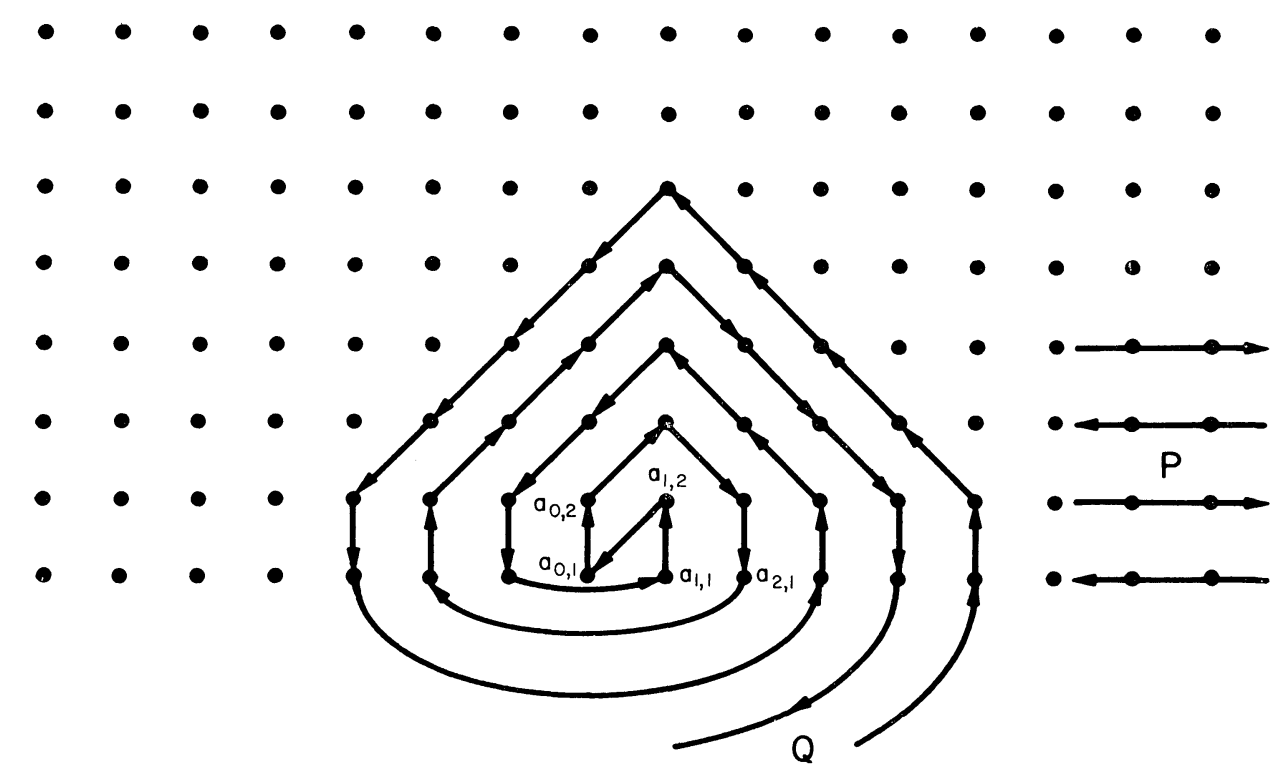

FIGURE 3

\section{REFERENCES}

1. E. A. Bertram, Even permutations as a product of two conjugate cycles, (to appear in J. Combinatorial Theory). 
2. A. B. Gray, In finite symmetric groups and monomial groups, Doctoral Dissertation, New Mexico State University, Las Cruces, New Mexico, 1960.

3. D. H. Husemoller, Ramified coverings of Riemann surfaces, Duke Math. J., 29 (1962), $167-174$.

4. O. Ore, Some remarks on commutators, Proc. Amer. Math. Soc., 2 (1951), 307-314. 5. M. A. Perles, Private communication.

Received November 10, 1970. This paper contains part of the author's doctoral dissertation, written under the direction of Professor T. S. Motzkin, at the University of California, Los Angeles.

UNIVERSITY OF HAWAII 


\title{
PACIFIC JOURNAL OF MATHEMATICS
}

\author{
EDITORS
}

\author{
H. SAMELSON \\ Stanford University \\ Stanford, California 94305

\section{R. HOBBY} \\ University of Washington \\ Seattle, Washington 98105
}

J. DUGUNDJI

Department of Mathematics

University of Southern California

Los Angeles, California 90007

RICHARD ARENS

University of California

Los Angeles, California 90024

\section{ASSOCIATE EDITORS}
E. F. BECKENBACH
B. H. NeUmanN
F. WoLF
K. YoshidA

\section{SUPPORTING INSTITUTIONS}

\author{
UNIVERSITY OF BRITISH COLUMBIA \\ CALIFORNIA INSTITUTE OF TECHNOLOGY \\ UNIVERSITY OF CALIFORNIA \\ MONTANA STATE UNIVERSITY \\ UNIVERSITY OF NEVADA \\ NEW MEXICO STATE UNIVERSITY \\ OREGON STATE UNIVERSITY \\ UNIVERSITY OF OREGON \\ OSAKA UNIVERSITY \\ UNIVERSITY OF SOUTHERN CALIFORNIA
}

\author{
STANFORD UNIVERSITY \\ UNIVERSITY OF TOKYO \\ UNIVERSITY OF UTAH \\ WASHINGTON STATE UNIVERSITY \\ UNIVERSITY OF WASHINGTON \\ $* * * *{ }^{*}$
AMERICAN MATHEMATICAL SOCIETY
CHEVRON RESEARCH CORPORATION
NAVAL WEAPONS CENTER
}

The Supporting Institutions listed above contribute to the cost of publication of this Journal, but they are not owners or publishers and have no responsibility for its content or policies.

Mathematical papers intended for publication in the Pacific Journal of Mathematics should be in typed form or offset-reproduced, (not dittoed), double spaced with large margins. Underline Greek letters in red, German in green, and script in blue. The first paragraph or two must be capable of being used separately as a synopsis of the entire paper. The editorial "we" must not be used in the synopsis, and items of the bibliography should not be cited there unless absolutely necessary, in which case they must be identified by author and Journal, rather than by item number. Manuscripts, in duplicate if possible, may be sent to any one of the four editors. Please classify according to the scheme of Math. Rev. Index to Vol. 39. All other communications to the editors should be addressed to the managing editor, Richard Arens, University of California, Los Angeles, California, 90024.

50 reprints are provided free for each article; additional copies may be obtained at cost in multiples of 50 .

The Pacific Journal of Mathematics is published monthly. Effective with Volume 16 the price per volume (3 numbers) is $\$ 8.00$; single issues, $\$ 3.00$. Special price for current issues to individual faculty members of supporting institutions and to individual members of the American Mathematical Society: $\$ 4.00$ per volume; single issues $\$ 1.50$. Back numbers are available.

Subscriptions, orders for back numbers, and changes of address should be sent to Pacific Journal of Mathematics, 103 Highland Boulevard, Berkeley, California, 94708.

PUBLISHED BY PACIFIC JOURNAL OF MATHEMATICS, A NON-PROFIT CORPORATION

Printed at Kokusai Bunken Insatsusha (International Academic Printing Co., Ltd.), 270, 3chome Totsuka-cho, Shinjuku-ku, Tokyo 160, Japan. 


\section{Pacific Journal of Mathematics}

Vol. 39, No. $2 \quad$ June, 1971

Edward Arthur Bertram, Permutations as products of conjugate infinite

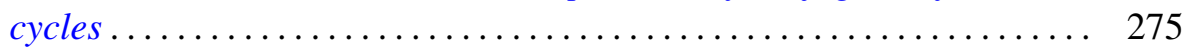

David Blair, Almost contact manifolds with Killing structure tensors ...... 285

Bruce Donald Calvert, Nonlinear equations of evolution ............. 293

Bohumil Cenkl and Giuliano Sorani, Cohomology groups associated with



Martin Aaron Golubitsky and Bruce Lee Rothschild, Primitive subalgebras

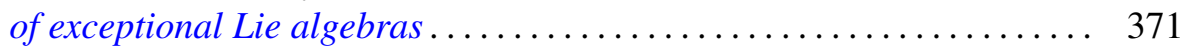

Thomas J. Jech, Two remarks on elementary embeddings of the universe ... 395

Harold H. Johnson, Conditions for isomorphism in partial differential equations........................................ 401

Solomon Leader, Measures on semilattices ..................... 407

Donald Steven Passman, Group rings satisfying a polynomial identity. II .......................................... 425

Ralph Tyrrell Rockafellar, Integrals which are convex functionals. II . . . . . 439

Stanisław Sławomir Świerczkowski, Cohomology of group germs and Lie

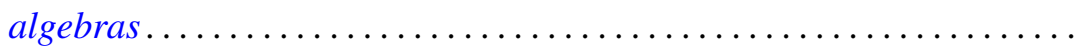

John Griggs Thompson, Nonsolvable finite groups all of whose local subgroups are solvable. III ............................ 483

Alan Curtiss Tucker, Matrix characterizations of circular-arc graphs .... 535 